1 Hacettepe Journal of Mathematics and Statistics

$\bigcap$ Volume 47 (2) (2018), 261-271

\title{
Coefficient inequalities for Janowski-Sakaguchi type functions associated with conic regions
}

\author{
Muhammad Arif*, Zhi-Gang Wang ${ }^{\dagger \ddagger}$, Rafiullah Khan ${ }^{\S}$ and See Keong Lee
}

\begin{abstract}
The purpose of the present paper is to introduce and study some new subclasses of Sakaguchi-type functions defined by using the concept of Janowski functions in conic regions. Various interesting properties such as sufficiency criteria, coefficient estimates and distortion result are investigated for these function classes.
\end{abstract}

Keywords: Sakaguchi-type function; Janowski function; conic domain.

2000 AMS Classification: Primary 30C45; Secondary 30C50.

Received: 01.12.2016 Accepted: 31.01.2017 Doi: 10.15672/HJMS.2017.448

\section{Introduction}

Let $\mathcal{A}$ be the class of functions $f$ of the form

$$
f(z)=z+\sum_{n=2}^{\infty} a_{n} z^{n},
$$

which are analytic in the open unit disk $\mathbb{D}=\{z \in \mathbb{C}:|z|<1\}$. As usual, $\mathcal{S}$ represents the class of all functions in $\mathcal{A}$ which are univalent in $\mathbb{D}$.

If $f$ and $g$ are analytic functions in $\mathbb{D}$, then we say that $f$ is subordinate to $g$, denoted by $f \prec g$ or $f(z) \prec g(z)$, if there exists an analytic function $w$ in $\mathbb{D}$ with $|w(z)|<|z|$

*Department of Mathematics, Abdul Wali Khan University Mardan, 23200 Mardan, Pakistan. Email : marifmaths@awkum.edu.pk

${ }^{\dagger}$ School of Mathematics and Computing Science, Hunan First Normal University, Changsha 410205, Hunan, China.

Email : wangmath@163.com

${ }^{\ddagger}$ Corresponding Author.

${ }^{\S}$ Department of Mathematics, Abdul Wali Khan University Mardan, 23200 Mardan, Pakistan. Email : rafilec@gmail.com

${ }^{\top}$ School of Mathematical Sciences, Universiti Sains Malaysia, 11800 USM, Penang, Malaysia. Email : sklee@cs.usm.my 
such that $f(z)=g(w(z))$. Furthermore, if the function $g$ is univalent in $\mathbb{D}$, then we have the following equivalence:

$$
f(z) \prec g(z) \Longleftrightarrow f(0)=g(0) \text { and } f(\mathbb{D}) \subset g(\mathbb{D}) .
$$

For arbitrary fixed numbers $A, B$ and $\sigma$ satisfying $-1 \leq B<A \leq 1,0 \leq \sigma<1$, let $\mathcal{P}(A, B, \sigma)$ denote the family of functions

$$
q(z)=1+\sum_{n=1}^{\infty} q_{n} z^{n} \quad(z \in \mathbb{D}) .
$$

The function $q$ belongs to the class $\mathcal{P}(A, B, \sigma)$ if and only if

$$
q(z) \prec(1-\sigma)\left(\frac{1+A z}{1+B z}\right)+\sigma .
$$

Thus, $q \in \mathcal{P}(A, B, \sigma)$ if and only if for some $w$ with $|w(z)|<|z|$, we have

$$
q(z)=\frac{(1-\sigma)(1+A w(z))+\sigma(1+B w(z))}{(1+B w(z))} .
$$

This class was investigated by Polatoglu [18], and further by putting $\sigma=0$ in $\mathcal{P}(A, B, \sigma)$, we get the class $\mathcal{P}(A, B)$ intoduced by Janowski [6]. Also we note that $\mathcal{P}(1,-1, \sigma) \equiv \mathcal{P}(\sigma)$ denotes the familiar class of functions with positive real part greater than $\sigma$ (see, for details, [4]). The classes $\mathcal{P}$ and $\mathcal{P}[A, B]$ are connected by

$$
p(z) \in \mathcal{P}(\sigma) \Longleftrightarrow \frac{(A+1) p(z)-(A-1)}{(B+1) p(z)-(B-1)} \in \mathcal{P}[A, B, \sigma] .
$$

Janowski functions are studied by several researchers like Cho [2], Liu and Noor [10], Liu and Srivastava [12], Noor and Arif [13], Polatoğlu [17], Shams et al. [21] and Wang et al. $[24]$, see also $[3,11]$.

Consider the domain

$$
\Omega_{k}=\left\{u+i v: u>k \sqrt{(u-1)^{2}+v^{2}}\right\},
$$

for fixed $k, \Omega_{k}$ represents the conic region bounded by the imaginary axis $(k=0)$, the right branch of a hyperbola $(0<k<1)$ and a parabola $(k=1)$ and an ellipse $(k>1)$. Also, we note that, for no choice of $k(k>1), \Omega_{k}$ reduces to a disk. This domain was studied by Kanas [7] and Kanas and Wisniowska [8, 9]. They also extended this domain to $\Omega_{k, \sigma}$ defined by

$$
\Omega_{k, \sigma}=(1-\sigma) \Omega_{k}+\sigma \quad(0 \leq \sigma<1) .
$$

The function $p_{k, \sigma}$, with $p_{k, \sigma}(0)=1, p_{k, \sigma}^{\prime}(0)>0$ plays the role of extremal for these conic domain $\Omega_{k, \sigma}$ and is given by

$$
p_{k, \sigma}(z)=\left\{\begin{array}{cc}
\frac{1+(1-2 \sigma) z}{1-z}, & k=0, \\
1+\frac{2(1-\sigma)}{\pi^{2}}\left(\log \frac{1+\sqrt{z}}{1-\sqrt{z}}\right)^{2}, & k=1, \\
1+\frac{2(1-\sigma)}{1-k^{2}} \sinh ^{2}\left[\left(\frac{2}{\pi} \arccos k\right) \operatorname{arctanh} \sqrt{z}\right], & 0<k<1, \\
1+\frac{(1-\sigma)}{k^{2}-1} \sin \left(\frac{\pi}{2 R(t)} \int_{0}^{\frac{u(z)}{\sqrt{t}}} \frac{1}{\sqrt{1-x^{2}} \sqrt{1-(t x)^{2}}} \mathrm{~d} x\right)+\frac{1}{k^{2}-1}, & k>1,
\end{array}\right.
$$


where $u(z)=\frac{z-\sqrt{t}}{1-\sqrt{t z}}(t \in(0,1))$ and $t$ is chosen such that $k=\cosh \left(\frac{\pi R^{\prime}(t)}{4 R(t)}\right)$, with $R(t)$ is Legendre's complete elliptic integral of the first kind and $R^{\prime}(t)$ is complementary integral of $R(t)$. For details on related work about conic domains, see [1, 5, 8, 9, 14, 23].

Let $\mathcal{P}_{p_{k, \sigma}}$ denote the class of all functions $p$ which are analytic in $\mathbb{D}$ with $p(0)=1$ and $p(z) \prec p_{k, \sigma}(z)$ for $z \in \mathbb{D}$. Clearly, it can be seen that $\mathcal{P}_{p_{k, \sigma}} \subset \mathcal{P}$.

1.1. Definition. (See [15]) A function $p \in k-\mathcal{P}[A, B]$ if and only if

$$
p(z) \prec \frac{(A+1) p_{k}(z)-(A-1)}{(B+1) p_{k}(z)-(B-1)} \quad(k \geq 0),
$$

where $p_{k}(z)=p_{k, 0}(z)$ and $-1 \leq B<A \leq 1$.

Geometrically, the function $p \in k-\mathcal{P}[A, B]$ takes all values in conic domain $\Omega_{k}[A, B],-1 \leq$ $B<A \leq 1, k \geq 0$ such that for $w \in \Omega_{k}[A, B]$, we have

$$
\Re\left(\frac{(B-1) w(z)-(A-1)}{(B+1) w(z)-(A+1)}\right)>k\left|\frac{(B-1) w(z)-(A-1)}{(B+1) w(z)-(A+1)}-1\right|,
$$

or equivalently,

$$
\begin{aligned}
\Omega_{k}[A, B]= & \left\{u+i v:\left[\left(B^{2}-1\right)\left(u^{2}+v^{2}\right)-2(A B-1) u+\left(A^{2}-1\right)\right]^{2}\right. \\
> & k^{2}\left[-2(B+1)\left(u^{2}+v^{2}\right)+2(A+B+2) u-2(A+1)^{2}\right. \\
& \left.\left.+4(A-B)^{2} v^{2}\right]\right\} .
\end{aligned}
$$

The domain $\Omega_{k}[A, B]$ contains the conic domain $\Omega_{k}$ inside the circular region defined by $\Omega[A, B]$. The impact of $\Omega[A, B]$ on the conic domain $\Omega_{k}$ changes the original shape of the conic regions. The ends of hyperbola and parabola get closer to each other but never meet anywhere and the ellipse gets the shape of oval. When $A=1, B=-1$, the radius of the circular disk defined by $\Omega[A, B]$ tends to infinity, consequently the arms of hyperbola and parabola expand and the oval turns into ellipse.

Motivated essentially by the recent paper of Noor and Malik [15], we define some classes of analytic functions associated with conic domains as follows:

1.2. Definition. A function $p \in k-\mathcal{P}[A, B, \sigma]$ if and only if

$$
p(z) \prec \frac{(A+1) p_{k, \sigma}(z)-(A-1)}{(B+1) p_{k, \sigma}(z)-(B-1)},
$$

where $p_{k, \sigma}(z)$ is defined by (1.3) and $0 \leq \sigma<1, k \geq 0$ and $-1 \leq B<A \leq 1$.

1.3. Definition. Let $f \in \mathcal{A}$. Then $f \in k-\mathcal{U S}[A, B, \sigma, t]$ if and only if

$$
\frac{(1-t) z f^{\prime}(z)}{f(z)-f(t z)} \in k-\mathcal{P}[A, B, \sigma]
$$

for some $0 \leq \sigma<1,-1 \leq B<A \leq 1,|t| \leq 1, t \neq 1$ and $k \geq 0$.

1.4. Definition. Let $f \in \mathcal{A}$. Then $f \in k-\mathcal{U}[A, B, \sigma, t]$ if and only if

$$
\frac{(1-t)\left(z f^{\prime}(z)\right)^{\prime}}{f^{\prime}(z)-t f^{\prime}(t z)} \in k-\mathcal{P}[A, B, \sigma],
$$

for some $0 \leq \sigma<1,-1 \leq B<A \leq 1,|t| \leq 1, t \neq 1$ and $k \geq 0$.

It can easily be seen that

$$
f \in k-\mathcal{U}[A, B, \sigma, t] \Longleftrightarrow z f^{\prime} \in k-\mathcal{U S}[A, B, \sigma, t] .
$$

We here present several special cases of the above mentioned function classes. 
(1) For $\sigma=0$ and $t=0$ the above classes reduce to the classes $k-\mathcal{S T}[A, B]$ and $k-\mathcal{U C}[A, B]$ studied recently by Noor and Malik [15];

(2) For $k=0, A=1, B=-1$, we obtain the classes $\mathcal{S}(\sigma, t)$ and $\mathcal{T}(\sigma, t)$ discussed in $[16]$;

(3) The classes $0-\mathcal{U S}[1,-1, \sigma,-1]=\mathcal{S}(\sigma,-1)$ and $0-\mathcal{U C}[1,-1, \sigma,-1]=\mathcal{T}(\sigma,-1)$ were introduced and studied by Owa et al. [16];

(4) The classes $0-\mathcal{U S}[1,-1,0,-1]=\mathcal{S}(0,-1)$ and $0-\mathcal{U C}[1,-1,0,-1]=\mathcal{T}(0,-1)$ were defined by Sakaguchi [20];

(5) The classes $0-\mathcal{S T}[A, B]=\mathcal{S}^{*}[A, B]$ and $0-\mathcal{U} \mathcal{C}[A, B]=\mathcal{C}[A, B]$, were introduced and studied by Janowski [6].

Unless otherwise mentioned, throughout this paper, we assume that

$$
0 \leq \sigma<1,-1 \leq B<A \leq 1,|t| \leq 1, t \neq 1 \text { and } k \geq 0 .
$$

The main objective of the present paper is to introduce and study some new subclasses of Sakaguchi-type functions defined by using the concept of Janowski functions in conic regions. Various interesting properties such as sufficiency criteria, coefficient estimates and distortion result are investigated for these function classes.

\section{Main Results}

To prove our main results, we need the following two lemmas.

2.1. Lemma. (See [19]) Let

$$
p(z)=1+\sum_{n=1}^{\infty} p_{n} z^{n} \prec F(z)=1+\sum_{n=1}^{\infty} d_{n} z^{n} \quad(z \in \mathbb{D}) .
$$

If $F$ is univalent in $\mathbb{D}$ and $F(\mathbb{D})$ is convex, then

$$
\left|p_{n}\right| \leq\left|d_{1}\right| \quad(n \geq 1) \text {. }
$$

2.2. Lemma. (See [15]) Let $p(z)=1+\sum_{n=1}^{\infty} c_{n} z^{n} \in k-\mathcal{P}[A, B, \sigma]$. Then

$$
\left|c_{n}\right| \leq\left|\delta_{A B}\right| \text {, }
$$

where

$$
\left|\delta_{A B}\right|=\frac{(A-B)\left|\delta_{k, \sigma}\right|}{2},
$$

and

$$
\delta_{k, \sigma}= \begin{cases}\frac{8(1-\sigma)(\arccos k)^{2}}{\pi^{2}\left(1-k^{2}\right)}, & 0 \leq k<1, \\ \frac{8(1-\sigma)}{\pi^{2}}, & k=1 \\ \frac{\pi^{2}(1-\sigma)}{4 \sqrt{t}\left(k^{2}-1\right) R^{2}(t)(1+t)}, & k>1 .\end{cases}
$$

We begin by deriving the following result.

2.3. Theorem. Let $f \in k-\mathcal{U S}[A, B, \sigma, t]$. Then

$$
\left|a_{2}\right| \leq \frac{\left|\delta_{A B}\right|}{\left|2-u_{2}\right|}
$$

and for $n \geq 3$

$$
\left|a_{n}\right| \leq \frac{\left|\delta_{A B}\right|}{\left|v_{n}\right|}\left\{\begin{array}{c}
1+\left|\delta_{A B}\right| \sum_{j=2}^{n-1} \frac{\left|u_{j}\right|}{\left|v_{j}\right|}+\left|\delta_{A B}\right|^{2} \sum_{j_{2}>j_{1}}^{n-1} \sum_{j_{1}=2}^{n-2} \frac{\left|u_{j_{1}} u_{j_{2}}\right|}{\left|v_{j_{1}} v_{j_{2}}\right|}+ \\
\left|\delta_{A B}\right|^{3} \sum_{j_{3}>j_{2}}^{n-1} \sum_{j_{2}>j_{1}}^{n-2} \sum_{j_{1}=2}^{n-3} \frac{\left|u_{j_{1}} u_{j_{2}} u_{j_{3}}\right|}{\left|v_{j_{1}} v_{j_{2}} v_{j_{3}}\right|}+\cdots+\left|\delta_{A B}\right|^{n-2} \prod_{j=2}^{n-1} \frac{\left|u_{j}\right|}{\left|v_{j}\right|}
\end{array}\right\},
$$


where $\delta_{A B}$ is defined by (2.1) and

$$
u_{n}=1+t+t^{2}+\cdots+t^{n-1} \text { and } v_{n}=n-u_{n} .
$$

Proof. Consider

$$
(1-t) z f^{\prime}(z)=(f(z)-f(t z)) p(z),
$$

where $p \in k-\mathcal{P}[A, B, \sigma]$. Let $p(z)=1+\sum_{n=1}^{\infty} c_{n} z^{n}$. It follows from (2.4) that

$$
z+\sum_{n=2}^{\infty} n a_{n} z^{n}=\left(z+\sum_{n=2}^{\infty} u_{n} a_{n} z^{n}\right)\left(1+\sum_{n=1}^{\infty} c_{n} z^{n}\right)
$$

Equating the coefficients of $z^{n}$ on both sides of the above equation, we have

$$
n a_{n}=u_{n} a_{n}+\sum_{j=1}^{n-1} a_{n-j} u_{n-j} c_{j}
$$

which implies that

$$
\left|n-u_{n}\right|\left|a_{n}\right| \leq \sum_{j=1}^{n-1}\left|u_{n-j}\right|\left|a_{n-j}\right|\left|c_{j}\right| .
$$

Since $p \in k-\mathcal{P}[A, B, \sigma]$, by Lemma 2.2 , we obtain

$$
\left|c_{n}\right| \leq\left|\delta_{A B}\right| \text {, }
$$

where $\delta_{A B}$ is defined by (2.1). Hence (2.5) reduces to

$$
\left|a_{n}\right| \leq \frac{\left|\delta_{A B}\right|}{\left|n-u_{n}\right|} \sum_{j=1}^{n-1}\left|u_{n-j}\right|\left|a_{n-j}\right| \quad(n \geq 2)
$$

It is clear from (2.6) that for $n \geq 2$, we see that

and

$$
\begin{aligned}
\left|a_{2}\right| & \leq \frac{\left|\delta_{A B}\right|}{\left|2-u_{2}\right|} \\
\left|a_{3}\right| & \leq \frac{\left|\delta_{A B}\right|}{\left|3-u_{3}\right|}\left[1+\left|\delta_{A B}\right| \frac{\left|u_{2}\right|}{\left|2-u_{2}\right|}\right]
\end{aligned}
$$

$$
\left|a_{4}\right| \leq \frac{\left|\delta_{A B}\right|}{\left|4-u_{4}\right|}\left[1+\left|\delta_{A B}\right|\left\{\frac{\left|u_{2}\right|}{\left|2-u_{2}\right|}+\frac{\left|u_{3}\right|}{\left|3-u_{3}\right|}\right\}+\left|\delta_{A B}\right|^{2}\left\{\frac{\left|u_{2}\right|\left|u_{3}\right|}{\left|2-u_{2}\right|\left|3-u_{3}\right|}\right\}\right] .
$$

Hence by using mathematical induction, we obtain the desired result.

For $t=0$ and $\sigma=0$, we get the following result obtained in [15].

2.4. Corollary. Let $f \in k-\mathcal{S T}[A, B]$. Then

$$
\left|a_{n}\right| \leq \prod_{j=0}^{n-2} \frac{\left|\delta_{k}(A-B)-2 j B\right|}{2(j+1)} \quad(n \geq 2) .
$$

Furthermore, by taking $A=1$ and $B=-1$ in the last Corollary 2.4, we obtain the following result for the class $k-\mathcal{S T}$, which was introduced by Kanas and Wisniowska [8].

2.5. Corollary. Let $f \in k-\mathcal{S T}$. Then

$$
\left|a_{n}\right| \leq \prod_{j=0}^{n-2} \frac{\left|\delta_{k}+j\right|}{(j+1)} \quad(n \geq 2)
$$


By putting $A=1-2 \beta, 0 \leq \beta<1, B=-1, t=0$ and $\sigma=0$ in Theorem 2.3, we obtain the coefficient bounds for $\mathcal{S D}(k, \beta)$, defined by Shams et al. [21].

2.6. Corollary. Let $f \in \mathcal{S D}(k, \beta)$. Then

$$
\left|a_{n}\right| \leq \prod_{j=0}^{n-2} \frac{\left|\delta_{k}(1-\beta)+j\right|}{(j+1)} \quad(n \geq 2)
$$

The inequality (2.7) is better than the result obtained by Owa et al. [16].

By setting $t=0, \sigma=0, k=0$ and $\delta_{k}=2$ in Theorem 2.3, we get the following coefficient bounds which was proved by Janowski [6].

2.7. Corollary. Let $f \in \mathcal{S}^{*}[A, B]$. Then

$$
\left|a_{n}\right| \leq \prod_{j=0}^{n-2} \frac{|(A-B)-j B|}{(j+1)} \quad(n \geq 2)
$$

Further by setting $A=1-2 \beta$ with $0 \leq \beta<1, B=-1$, we obatin the coefficient estimates for the class $\mathcal{S}^{*}(\beta)$, introduced by Robertson [4].

2.8. Theorem. Let $f \in k-\mathcal{U}[A, B, \sigma, t]$. Then, for $n \geq 2$

$$
\left|a_{n}\right| \leq \frac{\left|\delta_{A B}\right|}{n\left|v_{n}\right|}\left\{\begin{array}{c}
1+\left|\delta_{A B}\right| \sum_{j=2}^{n-1} \frac{\left|u_{j}\right|}{\left|v_{j}\right|}+\left|\delta_{A B}\right|^{2} \sum_{j_{2}>j_{1}}^{n-1} \sum_{j_{1}=2}^{n-2} \frac{\left|u_{j_{1}} u_{j_{2}}\right|}{\left|v_{j_{1}} v_{i_{2}}\right|} \\
+\left|\delta_{A B}\right|^{3} \sum_{j_{3}>j_{2}}^{n-1} \sum_{j_{2}>j_{1}}^{n-2} \sum_{j_{1}=2}^{n-3} \frac{\left|u_{j_{1}} u_{j_{2}} u_{j_{3}}\right|}{\left|v_{j_{1}} v_{j_{2}} v_{j_{3}}\right|}+\cdots\left|\delta_{A B}\right|^{n-2} \prod_{j=2}^{n-1} \frac{\left|u_{j}\right|}{\left|v_{j}\right|}
\end{array}\right\},
$$

where $\delta_{A B}, u_{n}$ and $v_{n}$ are defined by (2.1) and (2.3), respectively.

Proof. By virtue of Theorem 2.3 and the relationship (1.6), we get the required result.

2.9. Theorem. Let $f \in \mathcal{A}$ satisfies the condition

$$
\sum_{n=2}^{\infty}\left[\frac{2(k+1)}{(1-\sigma)}\left|n-u_{n}\right|+\left|n(B+1)-(A+1) u_{n}\right|\right]\left|a_{n}\right|<(A-B) .
$$

Then $f \in k-\mathcal{U S}[A, B, \sigma, t]$. 
Proof. Assume that (2.8) holds. Let us consider

$$
\begin{aligned}
& k\left|\frac{(B-1) \frac{(1-t) z f^{\prime}(z)}{f(z)-f(t)}-(A-1)}{(B+1) \frac{(1-t) z f^{\prime}(z)}{f(z)-f(t)}-(A+1)}-1\right| \\
= & k\left|\frac{(B-1)(1-t) z f^{\prime}(z)-(A-1)(f(z)-f(t z))}{(B+1)(1-t) z f^{\prime}(z)-(A+1)(f(z)-f(t z))}-1\right| \\
= & 2 k\left|\frac{(1-t) z f^{\prime}(z)-(f(z)-f(t z))}{(B+1)(1-t) z f^{\prime}(z)-(A+1)(f(z)-f(t z))}\right| \\
= & 2 k\left|\frac{(B-A) z+\sum_{n=2}^{\infty}\left(n(B+1)-(A+1) u_{n}\right) a_{n} z^{n}}{\left(B-u_{n}\right) a_{n} z^{n}}\right| \\
\leq & \frac{2 k \sum_{n=2}^{\infty}\left|\left(n-u_{n}\right) a_{n}\right|\left|z^{n}\right|}{(A-B)|z|-\sum_{n=2}^{\infty}\left|n(B+1)-(A+1) u_{n}\right|\left|a_{n}\right|\left|z^{n}\right|} \\
\leq & \frac{2 k \sum_{n=2}^{\infty}\left|\left(n-u_{n}\right) a_{n}\right|}{(A-B)-\sum_{n=2}^{\infty}\left|n(B+1)-(A+1) u_{n}\right|\left|a_{n}\right|},
\end{aligned}
$$

it follows from (2.8) that

$$
(A-B)-\sum_{n=2}^{\infty}\left|n(B+1)-(A+1) u_{n}\right|\left|a_{n}\right|>0 .
$$

To show that $f \in k-\mathcal{U S}[A, B, \sigma, t]$, it is sufficient to show that

$$
k\left|\frac{(B-1) \frac{(1-t) z f^{\prime}(z)}{f(z)-f(t z)}-(A-1)}{(B+1) \frac{(1-t) z f^{\prime}(z)}{f(z)-f(t z)}-(A+1)}-1\right|-\Re\left(\frac{(B-1) \frac{(1-t) z f^{\prime}(z)}{f(z)-f(t z)}-(A-1)}{(B+1) \frac{(1-t) z f^{\prime}(z)}{f(z)-f(t z)}-(A+1)}-1\right)<1-\sigma .
$$

From (2.9), we obtain

$$
\begin{aligned}
& k\left|\frac{(B-1) \frac{2 z f^{\prime}(z)}{f(z)-f(-z)}-(A-1)}{(B+1) \frac{2 z f^{\prime}(z)}{f(z)-f(-z)}-(A+1)}-1\right|-\Re\left(\frac{(B-1) \frac{2 z f^{\prime}(z)}{f(z)-f(-z)}-(A-1)}{(B+1) \frac{2 z f^{\prime}(z)}{f(z)-f(-z)}-(A+1)}-1\right) \\
& \leq(k+1)\left|\frac{(B-1) \frac{2 z f^{\prime}(z)}{f(z)-f(t)}-(A-1)}{(B+1) \frac{2 z f^{\prime}(z)}{f(z)-f(t)}-(A+1)}-1\right| \\
& \leq \frac{2(k+1) \sum_{n=2}^{\infty}\left|\left(n-u_{n}\right) a_{n}\right|}{(A-B)-\sum_{n=2}^{\infty}\left|n(B+1)-(A+1) u_{n}\right|\left|a_{n}\right|} .
\end{aligned}
$$

Last inequality is bounded by $1-\sigma$ if

$$
2(k+1) \sum_{n=2}^{\infty}\left|\left(n-u_{n}\right) a_{n}\right|<(1-\sigma)\left((A-B)-\sum_{n=2}^{\infty}\left|n(B+1)-(A+1) u_{n}\right|\left|a_{n}\right|\right) .
$$

Hence, we have

$$
\sum_{n=2}^{\infty}\left[\frac{2(k+1)}{(1-\sigma)}\left|n-u_{n}\right|+\left|n(B+1)-(A+1) u_{n}\right|\right]\left|a_{n}\right|<(A-B) .
$$


This completes the proof.

Taking $t=0, \sigma=0, A=1$ and $B=-1$ in Theorem 2.9 , then we get the following known result, proved by Kanas and Wisniowska [8].

2.10. Corollary. If $f \in \mathcal{A}$ satisfies the condition

$$
\sum_{n=2}^{\infty}[n+k(n-1)]\left|a_{n}\right|<1 \quad(k \geq 0),
$$

then $f \in k-\mathcal{S T}$.

When we put $t=0, \sigma=0, A=1-2 \beta$ and $B=-1$ in the Theorem 2.9, we obtain the following result proved by Shams et al. [21].

2.11. Corollary. If $f \in \mathcal{A}$ satisfies the condition

$$
\sum_{n=2}^{\infty}[n(k+1)-(k+\beta)]\left|a_{n}\right|<1-\beta \quad(k \geq 0),
$$

then $f \in \mathcal{S D}(k, \beta)$.

Moreover, by taking $k=0$ in Corollary 2.11, we get the following known result proved by Silverman [22].

2.12. Corollary. If $f \in \mathcal{A}$ satisfies the condition

$$
\sum_{n=2}^{\infty}[n-\beta]\left|a_{n}\right|<1-\beta \quad(0 \leq \beta<1),
$$

then $f \in \mathcal{S}^{*}(\beta)$.

2.13. Theorem. If $f \in \mathcal{A}$ satisfies the condition

$$
\sum_{n=2}^{\infty} n\left[\frac{2(k+1)}{(1-\sigma)}\left|n-u_{n}\right|+\left|n(B+1)-(A+1) u_{n}\right|\right]\left|a_{n}\right|<(A-B),
$$

then $f \in k-\mathcal{U C}[A, B, \sigma, t]$.

Proof. The proof follows directly by Theorem 2.9 and (1.6).

When $t=0$ and $\sigma=0$, we obtain the following result proved in [15].

2.14. Corollary. If $f \in \mathcal{A}$ satisfies the condition

$$
\sum_{n=2}^{\infty} n[2(k+1)(n-1)+|n(B+1)-(A+1)|]\left|a_{n}\right| \leq(A-B),
$$

then $f \in k-\mathcal{U C}[A, B]$.

We now define the classes $k-\mathcal{U} \mathcal{S}^{*}[A, B, \sigma, t]$ and $k-\mathcal{U} \mathcal{C}^{*}[A, B, \sigma, t]$ :

$$
k-\mathcal{U S} \mathcal{S}^{*}[A, B, \sigma, t]=\{f \in \mathcal{A}: f \text { satisfies the condition }(2.8)\},
$$

and

$k-\mathcal{U} \mathcal{C}^{*}[A, B, \sigma, t]=\{f \in \mathcal{A}: f$ satisfies the condition $(2.10)\}$. 
2.15. Theorem. If $f \in k-\mathcal{U} \mathcal{S}^{*}[A, B, \sigma, t]$, then

$$
|z|-\sum_{n=2}^{\infty}\left|a_{n}\right||z|^{n}-A_{l}|z|^{l+1} \leq|f(z)| \leq|z|+\sum_{n=2}^{\infty}\left|a_{n}\right||z|^{n}+A_{l}|z|^{l+1},
$$

where

$$
A_{l}=\frac{(1-\sigma)(A-B)-\sum_{n=2}^{l}\left[2(k+1)\left|n-u_{n}\right|+(1-\sigma)\left|(A+1) u_{n}-n(B+1)\right|\right]\left|a_{n}\right|}{\left\{[2(k+1)-(1-\sigma)(B+1)](l+1)+[(A+1)(1-\sigma)-2(k+1)]\left|u_{l+1}\right|\right\}} .
$$

Proof. From (2.8), we have

$$
\begin{aligned}
& \sum_{n=l+1}^{\infty}\left[2(k+1)\left|n-u_{n}\right|+(1-\sigma)\left|(A+1) u_{n}-n(B+1)\right|\right]\left|a_{n}\right| \\
\leq & (1-\sigma)(A-B)-\sum_{n=2}^{l}\left[2(k+1)\left|n-u_{n}\right|+(1-\sigma)\left|(A+1) u_{n}-n(B+1)\right|\right]\left|a_{n}\right| .
\end{aligned}
$$

Since

$$
\begin{aligned}
& {\left[2(k+1)\left|n-u_{n}\right|+(1-\sigma)\left|(A+1) u_{n}-n(B+1)\right|\right]} \\
& \geq[2(k+1)-(1-\sigma)(B+1)] n+[(A+1)(1-\sigma)-2(k+1)]\left|u_{n}\right|,
\end{aligned}
$$

and hence

$$
[2(k+1)-(1-\sigma)(B+1)] n+[(A+1)(1-\sigma)-2(k+1)]\left|u_{n}\right|
$$

is monotonically increasing with respect to $n$. Thus, from (2.11) and (2.12), we get

$$
\begin{aligned}
& \sum_{n=l+1}^{\infty}\left[[2(k+1)-(1-\sigma)(B+1)] n+[(A+1)(1-\sigma)-2(k+1)]\left|u_{n}\right|\right]\left|a_{n}\right| \\
& \leq(1-\sigma)(A-B)-\sum_{n=2}^{l}\left[2(k+1)\left|n-u_{n}\right|+(1-\sigma)\left|(A+1) u_{n}-n(B+1)\right|\right]\left|a_{n}\right| .
\end{aligned}
$$

Moreover, we see that

$$
\begin{aligned}
& \left\{[2(k+1)-(1-\sigma)(B+1)](l+1)+[(A+1)(1-\sigma)-2(k+1)]\left|u_{l+1}\right|\right\} \sum_{n=l+1}^{\infty}\left|a_{n}\right| \\
& \leq \sum_{n=l+1}^{\infty}\left\{[2(k+1)-(1-\sigma)(B+1)] n+[(A+1)(1-\sigma)-2(k+1)]\left|u_{n}\right|\right\}\left|a_{n}\right|
\end{aligned}
$$

From (2.13) and (2.14), we can write

$$
\sum_{n=l+1}^{\infty}\left|a_{n}\right| \leq A_{l}
$$

Therefore, we obtain

$$
|f(z)| \leq|z|+\sum_{n=2}^{l}\left|a_{n}\right||z|^{n}+\sum_{n=2}^{l}\left|a_{n}\right||z|^{l+1} \leq|z|+\sum_{n=2}^{l}\left|a_{n}\right||z|^{n}+A_{l}|z|^{l+1}
$$


and

$$
|f(z)| \geq|z|-\sum_{n=2}^{l}\left|a_{n}\right||z|^{n}-A_{l}|z|^{l+1}
$$

This completes the proof.

Similarly, we get the following result.

2.16. Theorem. If $f \in k-\mathcal{U C}^{*}[A, B, \sigma, t]$, then

$$
|z|-\sum_{n=2}^{l}\left|a_{n}\right||z|^{n}-B_{l}|z|^{l+1} \leq|f(z)| \leq|z|+\sum_{n=2}^{l}\left|a_{n}\right||z|^{n}+B_{l}|z|^{l+1}
$$

and

$$
1-\sum_{n=2}^{l} n\left|a_{n}\right||z|^{n-1}-C_{l}|z|^{l} \leq\left|f^{\prime}(z)\right| \leq 1+\sum_{n=2}^{l} n\left|a_{n}\right||z|^{n-1}+C_{l}|z|^{l}
$$

where

$$
B_{l}=\frac{(1-\sigma)(A-B)-\sum_{n=2}^{l} n\left[2(k+1)\left|n-u_{n}\right|+(1-\sigma)\left|n(B+1)-(A+1) u_{n}\right|\right]\left|a_{n}\right|}{(l+1)\left\{[2(k+1)-(1-\sigma)(B+1)](l+1)+[(A+1)(1-\sigma)-2(k+1)]\left|u_{l+1}\right|\right\}}
$$

and

$$
C_{l}=\frac{(1-\sigma)(A-B)-\sum_{n=2}^{l} n\left[2(k+1)\left|n-u_{n}\right|+(1-\sigma)\left|n(B+1)-(A+1) u_{n}\right|\right]\left|a_{n}\right|}{\left\{[2(k+1)-(1-\sigma)(B+1)](l+1)+[(A+1)(1-\sigma)-2(k+1)]\left|u_{l+1}\right|\right\}} .
$$

\section{ACKNOWLEDGMEnts}

The present investigation was supported by the Natural Science Foundation of Hunan Province under Grant no. 2016JJ2036.

\section{References}

[1] M. Arif, S. Mehmood, J. Sokól and J. Dziok, New subclass of analytic functions in conical domain associated with a linear operator, Acta Math. Sci., 36B (2016), 704-7016.

[2] N. E. Cho, The Noor integral operator and strongly close-to-convex functions, J. Math. Anal. Appl., 283 (2003), 202-212.

[3] N. E. Cho, O. S. Kwon and H. M. Srivastava, Inclusion relationships and argument properties for certain subclasses of multivalent functions associated with a family of linear operators, J. Math. Anal. Appl., 292 (2004), 470-480.

[4] A. W. Goodman, Univalent functions, Vol. I \& II, Polygonal Publishing House, Washington, New Jersey, 1983.

[5] W. Haq and S. Mahmood, Certain properties of a class of close-to-convex functions related to conic domains, Abstr. Appl. Anal., 2013 (2013), 6 pp.

[6] W. Janowski, Some extremal problems for certain families of analytic functions, Ann. Polon. Math., 28 (1973), 297-326.

[7] S. Kanas, Techniques of the differential subordination for domains bounded by conic sections, Int. J. Math. Math. Sci., 38 (2003), 2389-2400.

[8] S. Kanas and A. Wisniowska, Conic domains and starlike functions, Rev. Roumaine Math. Pures Appl., 45 (2000), 647-657.

[9] S. Kanas and A. Wisniowska, Conic regions and k-uniform convexity, J. Comput. Appl. Math., 105 (1999), 327-336. 
[10] J.-L. Liu and K. I. Noor, On subordinations for certain analytic functions associated with Noor integral operator, Appl. Math. Comput., 187 (2007), 1453-1460.

[11] J.-L. Liu and J. Patel, Certain properties of multivalent functions associated with an extended fractional integral operator, Appl. Math. Comput., 203 (2004), 703-713.

[12] J.-L. Liu and H. M. Srivastava, Certain properties of the Dziok-Srivastava operator, Appl. Math. Comput., 159 (2004), 485-493.

[13] K. I. Noor and M. Arif, Mapping properties of an integral operator, Appl. Math. Lett., 25 (2012), 1826-1829.

[14] K. I. Noor, M. Arif and W. Ul-Haq, On k-uniformly close-to-convex functions of complex order, Appl. Math. Comput., 215 (2009), 629-635.

[15] K. I. Noor and S. N. Malik, On coeffcient inequalities of functions associated with conic domains, Comput. Math. Appl., 62 (2011), 2209-2217.

[16] S. Owa, T. Sekine and R. Yamakawa, On Sakaguchi type functions, Appl. Math. Comput., 187 (2007), 356-361.

[17] Y. Polatoglu, Some results of analytic functions in the unit disc, Publ. Inst. Math. (Beograd) (N. S.), 78 (2005), 79-86.

[18] Y. Polatoglu, S. M. Bolcal and E. Yavuz, A study on the generalization of Janowski functions in the unit disc, Acta Math. Acad. Paedagog. Nyhazi. (N. S.), 22 (2006), 97-105.

[19] W. Rogosinski, On the coefficients of subordinate functions, Proc. Lodon Math. Soc., 48 (1943), 48-82.

[20] K. Sakaguchi, On a certain univalent mapping, J. Math. Soc. Japan, 11 (1959), 72-75.

[21] S. Shams, S. R. Kulkarni and J. M. Jahangiri, Classes of uniformly starlike and convex functions, Int. J. Math. Math. Sci., 55 (2004), 2959-2961.

[22] H. Silverman, Univalent functions with negative coefficients, Proc. Amer. Math. Soc., 51 (1975), 109-116.

[23] H. M. Srivastava, M. R. Khan and M. Arif, Some subclasses of close-to-convex mappings associated with conic regions, Appl. Math. Comput., 285 (2016), 94-102.

[24] Z.-G. Wang, M. Raza, M. Ayaz and M. Arif, On certain multivalent functions involving the generalized Srivastava-Attiya operator, J. Nonlinear Sci. Appl., 9 (2016), 6067-6076. 
